\title{
La construccionalización de la desiderativa no sea que + subjuntivo como Motivación de un acto de habla previo*
}

\section{The constructionalization of the optative clause no sea que + subjunctive as Motivation of the previous speech act}

\author{
Lorena Núñez Pinero \\ Universidad Complutense de Madrid
}

Resumen. En este artículo se ofrece un análisis de un tipo de construcción compuesta por dos oraciones: Trae el paraguas, no sea que llueva esta tarde y Trae el paraguas, no sea que se mojen los libros.

La segunda oración, encabezada por una negación y un verbo de suceso, era una desiderativa negativa que se construccionalizó como subordinada. En la nueva construcción la subordinada desempeña la función pragmática Motivación, es decir, el hablante justifica con ella el acto de habla anterior. El hablante no desea el cumplimiento del evento introducido por la oración subordinada y por ello busca un remedio o, si no tiene remedio, una forma de resistirse a ello. Así, la función pragmática Motivación se codifica morfosintácticamente, bien mediante una oración de interpretación final negativa, o bien mediante una oración de interpretación causal.

Palabras clave: desiderativa, construccionalización, Motivación, yuxtaposición, causa, finalidad

Abstract. This paper offers an analysis of a type of construction composed by two clauses: 'Bring the umbrella, in case it rains this afternoon' and 'Bring the umbrella, so the books don't get wet'.

Data de recepción: 11-05-2018 - Data de aceptación: 26-07-2018.

* Quiero expresar mi agradecimiento por sus correcciones y consejos a Silvia Iglesias Recuero y a Ignacio Bosque. 
The second clause, headed by a negation and an event verb, was a negative optative that was constructionalized as subordinate. In the new construction the subordinate clause fulfills the pragmatic function Motivation, that is, the speaker uses it to justify the previous speech act. The speaker does not want the occurrence of the event introduced by the subordinate clause and therefore seeks a remedy or, if there is no remedy, a way to cope with it. Thus, the pragmatic Motivation function is encoded morphosyntactically, either by a clause with a negative purpose reading, or by a clause with a causal reading.

Keywords: optative, juxtaposition, constructionalization, Motivation, cause, purpose

\section{INTRODUCCIÓN}

Oraciones como Trae el paraguas, no sea que llueva esta tarde o como Trae el paraguas, no sea que se mojen los libros no han recibido apenas atención por parte de los estudiosos, quizá porque están yuxtapuestas, es decir, no se explicita mediante una conjunción o una locución conjuntiva la relación entre ellas 1 .

El objetivo de este trabajo es llevar a cabo una investigación sobre sus orígenes y su naturaleza. Especialmente importante será descubrir qué relación existe entre la función pragmática de la segunda oración y la codificación morfosintáctica que se hace de ella, así como averiguar qué elementos inducen la interpretación causal, según veremos, en Trae el paraguas, no sea que llueva esta tarde, y final negativa, en Trae el paraguas, no sea que se mojen los libros.

Veremos cómo la segunda oración se subordina en el nivel de la ilocución a los enunciados que la preceden, que pueden ser de distintas modalidades, pero especialmente se subordina a enunciados imperativos. Nos centraremos en el estudio de la variante con verbo ser en presente de subjuntivo en la subordinada (no sea que...),

1 No es algo exclusivo de los estudios de la gramática del español la falta de atención a las oraciones yuxtapuestas: cf. Gohl (2000: 83-84). Por otro lado, este tipo de oraciones normalmente se ha asociado en la bibliografía al lenguaje infantil, a las clases bajas, al registro coloquial (al que Montolío 1999: 3678 adscribe nuestra construcción), a las fases pre- y proto-escriturales de una lengua, o a las lenguas «primitivas». Esta idea necesita ser revisada y contrastada mediante trabajos de corpus, como Cano Aguilar (2011: 480) recuerda en su investigación sobre la misma construcción que aquí analizamos, pero en un corpus del siglo XVI. La conclusión provisional que extrae de su corpus es la siguiente (2011: 488): «(...) puede hallarse en situaciones de diálogo, en tipos textuales con cierta tendencia a la imitación de la oralidad (los diálogos picarescos de Mateo Alemán o López de Úbeda, o en la comedia). Pero es mucho más habitual en tradiciones textuales más propias de la distancia comunicativa: exposiciones y elucubraciones cuasi ensayísticas, moralizantes, etc., es decir, en el discurso argumentativo y expositivo de la literatura ascética o de los memoriales políticos. Esa tendencia continuará en el XVII»). 
aunque en el apartado 2 examinaremos otras variantes de la construcción. El verbo de la subordinada es un verbo de suceso (ser) que introduce una subordinada completiva en función de sujeto.

En el apartado 3 se abordará su caracterización: las subordinadas de interpretación final negativa son siempre prospectivas, mientras que las de interpretación causal pueden ser prospectivas o no. También veremos qué relación puede haber entre el control del predicado y las dos interpretaciones ${ }^{2}$ y cómo opera de distinta manera la negación en las subordinadas de interpretación final y en las de interpretación causal. Independientemente de cuál sea la interpretación, la subordinada ofrece una Motivación, es decir, justifica la enunciación del acto de habla previo. Por otro lado, el cumplimiento del evento del acto de habla previo puede conceptualizarse como un remedio para que no se cumpla el evento significado por la subordinada: la interpretación de esta última es entonces final negativa. En cambio, si el cumplimiento del primer evento se conceptualiza como un remedio de las consecuencias derivadas del evento de la subordinada (puesto que el evento en sí se conceptualiza como incontrolable), la interpretación de la subordinada es causal.

En el apartado 4 descubriremos cómo la subordinada era en realidad una construcción desiderativa que sufrió, en combinación con la oración anterior, un proceso de construccionalización.

Por construccionalización entendemos, junto con Traugott y Trousdale (2013: 22), lo siguiente:

Constructionalization is the creation of form new $^{-m_{\text {eaning }}}$ (combinations of) signs. It forms new type nodes, which have new syntax or morphology and new coded meaning, in the linguistic network of a population of speakers. It is accompanied by changes in degree of schematicity, productivity, and compositionality. The constructionalization of schemas always results from a succession of micro-steps and is therefore gradual.

Hablamos de construccionalización y no de gramaticalización, porque la construcción que estamos estudiando se forma a partir de otra construcción consolidada en la lengua, más abstracta: la construcción desiderativa, de la que hereda, como veremos, muchas de sus propiedades. No se crea simplemente una categoría gramatical nueva, sino que se extiende el uso de un esquema preexistente a otros contextos y termina consolidándose como una nueva construcción más específica y más compleja sintácticamente:

Con «control» nos referiremos a una propiedad de un agente volitivo que implica capacidad para desencadenar la acción. 
The term "construction" regularly appears in the grammaticalization literature (see e.g. Lehmann 1985; Hopper \& Traugott 2003), but it is used there typically to refer to the surrounding context in which a particular expression comes to have a more grammatical function. The term is not used in such research to describe a form-meaning pairing, at any level of generality, which is shared across a group of speakers (i.e. a "conventional symbolic unit", the definition of a construction proposed by Langacker 1987 and Croft 2005, among others). The model of constructionalization as proposed by Traugott \& Trousdale (2013) takes the construction as the basic unit of language, and seeks to explore how new constructions come into being, and how they develop different functions. (Trousdale 2014: 559)

Por tanto, tomamos como unidad de estudio la construcción, porque nos encontramos ante un compuesto gramatical que pertenece tanto al nivel fonológico, como al semántico, al sintáctico y al pragmático, pues, como se irá viendo a lo largo de este artículo, las dos oraciones: 1) conforman una unidad con unas características sintácticas y semánticas determinadas; 2) tienen unas propiedades discursivas específicas y la función pragmática (Motivación) que desempeña la segunda oración es siempre la misma.

Esta unidad no se adecua, lamentablemente, a un modelo gramatical de análisis que no tome el contexto y la función comunicativa como elementos fundamentales de su sistema o que considere la oración la unidad máxima.

\section{LAS VARIANTES DE LA CONSTRUCCIÓN}

En RAE-ASALE (2009) es donde más información puede recopilarse sobre la construcción. En los párrafos $§ 24.1 \mathrm{~m}, \S 27.4 \mathrm{~g}$, $\$ 46.7 \mathrm{i}$ y $§ 46.7 \mathrm{k}$, concretamente, se recogen las variantes en función de cómo se introduzca la segunda oración. Se dice, por un lado, que existe una oposición no \{vaya fuera\} a ser que y que se escoge la forma vaya para expresar tiempo presente o futuro y la forma fuera cuando se expresa pasado (\$24.1m). Los ejemplos que ofrece Gómez Torrego (1988: 74) se ajustan a esta descripción:

(1) Se decidieron a entrar, no fuera a ser que los descubrieran.

(2) Entremos, no vaya a ser que nos descubran.

En (1) el tiempo de la segunda oración es pos-pretérito y en (2) significa futuro ${ }^{3}$.

Nos servimos en este artículo para referirnos a los tiempos de la terminología de Bello (1988 [1847]: 431-479). 
En 46.7i se señala como variante no + subjuntivo:

(3) Vamos poco a poco, no suceda que pisemos en tierra falsa (Torres Villarroel, Anatomía).

Por otro lado, se considera una variante propia del registro coloquial del español europeo $\{$ vaya $\sim$ fuera $\}$ a ser que sin negación:

(4) Además, no se atreve ni a bailar, vaya a ser que al día siguiente se vea en el periódico en semejante trance (Lindo, Tinto, apud §24.1m).

Así como la variante vaya que, que se aconseja evitar:

(5) Vaya que tengamos un disgusto (\$46.7i).

En cambio, cosa que + subjuntivo sin negación (frente a no sea cosa que) se documenta como una variante del registro coloquial propia de las áreas caribeña, andina y rioplatense:

(6) Y, cosa que [la huelga] no se haga más grande, el gobierno tuvo que aceptar sus peticiones (Viezzer, Hablar, apud §46.7k)4.

También se ejemplifica y se desaconseja el uso de la variante no siendo que:

(7) Ah, lo único es que se lo adviertas a papá, si se levanta, no siendo que se le ocurra entrar en el despacho y lo despierte (Martín Gaite, Fragmentos, apud \$27.4g).

Existen, según esto, las siguientes variantes de no sea que + subjuntivo: no + subjuntivo, no fuera a ser que, no vaya a ser que, fuera a ser que, vaya a ser que, no sea cosa que, cosa que y no siendo que.

Puede apreciarse cómo en ningún caso no hubiera se sido que encabeza la segunda oración. Esto puede explicarse porque esta segunda oración expresa, según iremos viendo, un temor, es decir, un evento posible: no puede emplearse, así, una forma propia de la contrafactualidad.

$4 \quad$ Parece, a la vista de los ejemplos que se ofrecen en RAE-ASALE (2009), que esta última variante de la construcción tiene un comportamiento claramente distinto, y una interpretación exclusivamente final. Sin embargo, los ejemplos de no \{sea / fuera\} cosa que que se aportan en $\S 46.7 \mathrm{k}$ tienen todos interpretación causal. El estudio de esta distribución sobrepasa los objetivos de este trabajo. 


\section{CARACTERIZACIÓN DE LA CONSTRUCCIÓN}

\subsection{Propiedades morfosintácticas: estructura, posiciones, dependencia y modalidad}

En este trabajo nos ceñiremos al análisis de la variante de la construcción en la que la segunda oración se introduce mediante no sea que.

Una propiedad suficiente de esta construcción, que sorprendentemente no se ha explicitado en la bibliografía, es la posposición obligatoria de la oración encabezada por no sea que:

(8) ??No sea que se mojen los libros, trae el paraguas.

(9) ??No sea que llueva esta tarde, trae el paraguas.

Este orden viene propiciado por la función pragmática (Motivación) que desempeñan las oraciones de no sea que, como veremos en el apartado 3.3.

La construcción está formada por dos oraciones yuxtapuestas. López García (1999: 3543), así como Gili Gaya (1943: 262-264), señalan que la yuxtaposición puede significar lo mismo que la coordinación o la subordinación, solo que ese significado se deduce entonces del contexto ${ }^{5}$. En el mismo sentido, Lehmann (1988: 210) dice lo siguiente:

the presence or absence of a connective device between two clauses has nothing to do with parataxis vs. hypotaxis, but is exclusively a question of syndesis. In particular, it is not the case that either the concept of hypotaxis or the concept of subordination require the use of a conjunction.

El análisis de una construcción formada por yuxtaposición entraña de por sí cierta dificultad, principalmente porque, al no contar con conjunciones, las relaciones que existen entre sus oraciones están más encubiertas. Los pocos lingüistas que se han fijado en la construcción que aquí estudiamos (Cano Aguilar 2011; RAE-ASALE 2009; Galán Rodríguez 1992, 1999; Montolío 1999; Pérez Saldanya 1999; Carrera de

Sin embargo, como apuntan Brucart y Gallego (2009: 140), con este tipo de enfoque se da por supuesto que las oraciones yuxtapuestas pertenecen al dominio del discurso y no al de la oración, por lo que no se llega nunca a proponer un análisis formal, solamente semántico. Ellos en realidad tampoco lo ofrecen: no se ocupan de este tipo de construcciones en el artículo, sino de aquellas en las que sí hay una conjunción. Su análisis de la segunda oración de las construcciones finales y de las causales como un SPrep cuya preposición rige una subordinada completiva no parece válido para esta construcción, que carece de conjunción. 
la Red 1989; Gómez Torrego 1988) coinciden en interpretar la segunda oración como subordinada a la primera, o al menos como dependiente conceptualmente de ella.

Sin embargo, aunque la segunda oración no puede existir sin la primera ${ }^{6}$ (y no a la inversa), no desempeña una función argumental en ella y no se puede conmutar por un adverbio, lo que hace posible, como veremos, que pueda funcionar en el nivel del discurso.

Otra cuestión fundamental, sin respuesta en la bibliografía, es si es posible yuxtaponer la oración de no sea que a una oración de cualquier modalidad o si hay alguna restricción ${ }^{7}$.

Sweetser (1990: 78), en una nota, se refiere a las restricciones de modalidad de las oraciones causales. De las que se integran en el nivel del discurso, como la de What are you doing tonight, because there's a good movie on, dice que pueden combinarse con modalidades distintas a la enunciativa, pero que las del nivel oracional, en cambio, solo pueden combinarse con otras modalidades si están «inside the scope» o, en el ejemplo anterior, 'dentro del alcance' de la interrogación. Sweetser no ofrece ejemplos de este tipo, pero parece que se refiere a casos como los siguientes, que Lapesa (1978: 183) incluye entre los de causales que son causa de la otra oración (del nivel oracional, por tanto):

(10) No te arrepientas porque te acusan.

(11) Arrepiéntete porque has causado daño a otros.

(12) Ojalá procedas así porque es tu deber y no por el dinero.

(13) ¡Lástima que protestaras porque estabas irritado y no por defender la justicia!

(14) ¿Cómo no te quejaste porque calumniaran a tus amigos?

En lo que respecta a nuestra construcción, la segunda oración (sea su interpretación causal o final negativa) se combina con gran frecuencia con la modalidad imperativa ${ }^{8}$ : esto es consecuencia, una vez más, del tipo de función pragmática (Motivación)

6 Así, parece más apropiado decir de la segunda oración que está subordinada, no que las dos oraciones establecen entre sí una relación de interdependencia o de interordinación (cf. Rojo 1978; Narbona 1990).

7 No obstante, en Cano Aguilar (2011: 483-486) se observa su frecuente aparición junto a oraciones imperativas y se menciona la posibilidad que tienen de combinarse con enunciativas.

8 En RAE-ASALE, 46.7i, se decía, al hablar de la variante encabezada por no + subjuntivo, que «adquiere sentido final en contextos imperativos y, en general, directivos, como en Dile algo, no te pase como la otra vez», pero pueden aducirse ejemplos que contradicen esta propiedad: de interpretación final negativa sin un contexto directivo: Traigo el paraguas, no se mojen los libros; y existen igualmente ejemplos en los que la primera oración tiene modalidad imperativa y la interpretación de la segunda no es final negativa, sino causal: Trae el paraguas, no llueva esta tarde. 
asociada a ella, que está a su vez relacionada con la amenaza que supone para la imagen del interlocutor un acto de habla directivo, como veremos en el apartado 3.3.

No obstante, en esta construcción es posible (al menos en teoría) combinar la segunda oración con cualquier modalidad en la primera, independientemente de que su interpretación sea final negativa o causal:

(15) Trae el paraguas, no sea que se mojen los libros.

(16) Traigo el paraguas, no sea que se mojen los libros.

(17) ¡Ojalá traiga el paraguas! No sea que se mojen los libros.

(18) ¿Te traes el paraguas? No sea que se mojen los libros.

(19) ¡Qué bien, un paraguas! No sea que se mojen los libros.

(20) Trae el paraguas, no sea que llueva esta tarde.

(21) Traigo el paraguas, no sea que llueva esta tarde.

(22) ¡Ojalá traiga el paraguas! No sea que llueva esta tarde.

(23) ¿Te traes el paraguas? No sea que llueva esta tarde.

(24) ¡Qué bien, un paraguas! No sea que llueva esta tarde.

La yuxtaposición no es el motivo de que funcionen esas combinaciones, pues también las paráfrasis con conjunción las admiten, como por ejemplo:

(25) ¿Te traes el paraguas? Para que no se mojen los libros.

Más bien se debe a que las oraciones de la construcción no guardan entre sí la misma relación que Me dijo y que ojalá trajera el paraguas en:

(26) Me dijo que ojalá trajera el paraguas.

En este último ejemplo que ojalá trajera el paraguas es un argumento del predicado de la principal, integrado en ella de manera que no solo no conserva ya su modalidad desiderativa, sino que además desempeña una función dentro de ella. En cambio, podemos observar cómo en los ejemplos anteriores (15-24) las segundas oraciones no son argumentos de las primeras. La entonación de coma o la pausa (según los casos) antes de la segunda oración también marca el menor grado de integración sintáctica entre las dos oraciones.

La oración subordinada está, así, menos integrada que una subordinada argumental, pero también, como veremos más adelante, menos integrada que una causal o una final del nivel de la proposición.

La combinatoria con otras modalidades refleja, por tanto, la falta de integración sintáctica de la oración de no sea que con la oración anterior y su funcionamiento en 
el nivel del discurso. Como veremos en los apartados 3.2 y 3.3, lo que hace la segunda oración solo puede entenderse como la causa o la finalidad del acto de habla anterior 9 .

Otras diferencias que suelen establecerse entre los complementos del nivel oracional y los del nivel del discurso no proceden aquí, ya que guardan relación con la existencia de conjunciones, ausentes de esta construcción: como, por ejemplo, la posibilidad de hacer una construcción dislocada con los complementos del nivel oracional o de recuperarlos mediante fóricos.

\subsection{Propiedades de la interpretación: causa o efecto, tiempos, control y negación}

Una cuestión fundamental que queda todavía por resolver es averiguar qué es lo que induce la interpretación final o causal en la construcción o, mejor dicho, cómo se conceptualiza la segunda oración de la construcción en unos casos como efecto y en otros como causa. Como causa, concretamente, potencial o hipotética.

El límite entre las oraciones causales y las finales no es muy nítido. De hecho, según la teoría aristotélica, en ambas se contiene una causa: «final» en las finales e «inicial» en las causales. Por eso es muy común que una misma conjunción o una misma preposición sirva para expresar los dos tipos de relación, como en español por: Lo hago por \{quedar bien / placer\}.

En ocasiones se ha catalogado la segunda oración de la construcción que aquí estudiamos como exclusivamente final (Galán Rodríguez 1992, 1999; Pérez Saldanya 1999; Carrera de la Red 1989; Gómez Torrego 1988), atendiendo solo a ejemplos del tipo Trae el paraguas, no sea que se mojen los libros y a su posible paráfrasis con para que no: Trae el paraguas para que no se mojen los libros. Sin embargo, puede apreciarse en ejemplos como Trae el paraguas, no sea que llueva esta tarde que esa paráfrasis con para que no no es siempre posible. En este caso solamente podría parafrasearse con por si (acaso) + indicativo: Trae el paraguas por si (acaso) llueve esta tarde.

9 Gohl (2000) estudia casos de causales asindéticas del alemán en la conversación que responden al acto de habla anterior, pero a menudo cada oración la pronuncia un hablante distinto y no constituyen construcciones como las que aquí estudiamos (con marcas como la negación + sea que y otras características que iremos viendo a lo largo del artículo). La autora estudia en su corpus con qué tipo de actos de habla se combinan las causales. La conexión lógica entre ellas se deduce pragmáticamente. En este sentido, véase el siguiente ejemplo del CNDHE (Sandoval 1604-1618, Historia de la vida y hechos del Emperador Carlos V): «Y diciendo esto, volvió a tomar con solos dos dedos el saquito, y al punto se le pasmaron, como si fueran de hierro, de tal manera que no pudo juntarlos ni tocarle con ellos. Quedaron los circunstantes admirados con el milagro, y la Madalena haciéndose lágrimas. Entonces Lucrecia, como quien adivinaba, dijo: - No sea que esté aquí el prepucio de Jesucristo, sobre el cual el Pontífice Clemente VII escribió a mi marido Juan Baptista». 
No obstante, algunos autores (Cano Aguilar 2011; RAE-ASALE 2009; Montolío 1999) sí se refieren a este tipo de ejemplos, parafraseables con por si, aunque ninguno de ellos alude en ningún momento a las propiedades de cada uno de los dos tipos de oración o a qué elementos inducen cada una de las interpretaciones. En RAEASALE (2009: §46.7j) se señala que no siempre la construcción admite la paráfrasis con para que + subjuntivo, sino que hay veces en que la paráfrasis adecuada es con por $s i+$ indicativo.

Cano Aguilar (2011) también ofrece ejemplos de no sea que + subjuntivo de interpretación causal y los considera «de finalidad secundaria».

Montolío (1999: 3678), por su parte, incluye este tipo de ejemplos entre las oraciones condicionales, aunque no hace referencia a la interpretación final negativa de la oración. Montolío incluye este tipo de construcciones entre las condicionales, porque sus correspondientes paráfrasis, las oraciones introducidas por por si o por si acaso también se han catalogado a veces como condicionales; otras veces, como causales; y otras, con etiquetas híbridas (cf. Núñez 1998 para el latín), ya que no son, según veremos, causales prototípicas, sino causales potenciales o hipotéticas.

También se ha señalado en la bibliografía (Galán Rodríguez 1992, 1999; Pérez Saldanya 1999), al hablar de la interpretación final, que la construcción guarda relación, lo mismo que las finales en general, con el llamado «subjuntivo volitivo».

Galán Rodríguez (1999: 3624) menciona la siguiente oración cuando habla de «oraciones finales de objetivo como deseo»: Voy a salir, no piensen que estoy enfermo, ejemplo que, según se ve, es semejante a Traigo el paraguas, no sea que se mojen los libros en el sentido de que sí permite la paráfrasis con para que. La definición que ofrece de la clase de las finales, digamos, «impuras», a la que pertenecen las «oraciones finales de objetivo como deseo», es la siguiente:

(...) se caracterizan en líneas generales por incumplir alguno de los dos rasgos básicos establecidos; esto es, o bien carecen de agente o bien alteran la relación de prospectividad y objetivo entre los términos A y B. Estas oraciones presentan el objetivo como utilidad, como consecuencia, como contraste o como deseo.

Previamente la autora ha establecido como propiedades de las finales puras la agentividad del sujeto de la principal y su correferencialidad con el de la subordinada.

A continuación, explica el «objetivo como deseo» como «algo no realizado sobre lo que el hablante vuelca su subjetividad», definición algo imprecisa que podría aplicarse, al menos, a cualquier tipo de final. La autora percibe que en ese ejemplo (Voy a salir, no piensen que estoy enfermo) la segunda oración guarda alguna relación con la modalidad desiderativa y, así, define esta clase de finales (entre las que incluye 
no solo construcciones yuxtapuestas de interpretación final como las que aquí se analizan, sino también otras introducidas por conjunciones) basándose en la definición más extendida en nuestra tradición de la modalidad desiderativa como 'expresión de deseos', que atiende exclusivamente a su semántica.

En Galán Rodríguez (1992: 145-146) también se señala la relación entre las oraciones finales y el subjuntivo que expresa deseo. Igualmente se hace en Pérez Saldanya (1999: 3308-3309):

El uso del subjuntivo en (...) contextos finales se justifica por el carácter volitivo o intencional que caracteriza la oración subordinada y, consiguientemente, porque esta oración no comunica ningún hecho sino un objetivo, un evento virtual, cuya realización, si llega a producirse, es necesariamente posterior a lo designado por la oración principal.

Enseguida veremos la relación que guarda esta construcción con la modalidad desiderativa.

En el apartado siguiente, en 3.3, se analiza la función del acto de habla expresado mediante la segunda oración y su relación con el acto de habla anterior. El primero se codifica mediante oraciones de interpretación causal o de interpretación final, que, en virtud de ciertas propiedades que las caracterizan (referidas al tiempo del evento, al control del predicado o a la distinta interpretación de la negación), sirven bien para ello. Algunas de esas propiedades, que a continuación examinaremos, las comparten con las construcciones desiderativas, que, como se verá en el apartado 4, están implicadas en el origen de la construcción que estamos estudiando, anteriores a los reanálisis que posibilitaron la creación de esta nueva construcción.

En las construcciones desiderativas ni el hablante ni el destinatario tienen control sobre el cumplimiento del evento significado en ellas. Esta característica tiene como consecuencia que presenten con gran frecuencia predicados inacusativos. En cambio, en las construcciones imperativas el destinatario sí tiene control sobre el evento pretendido $^{10}$. Los dos tipos de construcción pertenecen a la modalidad irrealis, que señala que el contenido no es real en el mundo en el que se habla.

10 Esta distinción pragmática es fundamental, ya que una desiderativa negada no se diferencia en español morfológicamente de una imperativa negada. Tampoco en latín: «synchronically it would be difficult to make a clear división between the two in Latin where the subjunctive mood is used for both. Each of these two construction types involve the speaker's desire, with the subjunctive mood serving to indicate that the situation described is generally considered a possibility and not actual reality» (Lakey 2015: 78). 
Un rasgo que suele diferenciar a las finales de las causales es la prospectividad ${ }^{11}$, es decir, las finales prototípicas significan un evento posterior al tiempo de referencia (el efecto), que en este caso es el tiempo del evento significado por la oración principal. Las causales prototípicas, en cambio, significan un evento anterior al tiempo de referencia (la causa) ${ }^{12}$.

Las oraciones de interpretación causal en la construcción que estamos estudiando no son prototípicas. Por ello pueden expresar un evento posterior al de la primera oración (Trae el paraguas, no sea que llueva esta tarde): pueden ser prospectivas. Este rasgo guarda relación con el origen desiderativo de esta construcción, como veremos en el apartado siguiente.

Sin embargo, las subordinadas de interpretación causal en la construcción no tienen por qué ser prospectivas:

(27) Debe acudir a la policía, señora, no sea que hayan matado a su marido.

En cambio, las finales negativas tienen una restricción temporal más fuerte: son siempre prospectivas, de tal manera que, si la segunda oración expresa tiempo presente (28) o antepresente (29), la interpretación es necesariamente causal:

(28) Debe acudir a la policía, señora, no sea que estén matando a su marido.

(29) Debe acudir a la policía, señora, no sea que su marido se haya perdido.

Esta distribución según los tiempos se encuentra reflejada en la construcción ya en el siglo XVII. Si tomamos los ejemplos que hay de ella en el Quijote de Cervantes $(1605,1615)$ y en el de Avellaneda (1614), en este caso en su variante de no + subjuntivo, podemos observar que, no por casualidad, en los casos de interpretación final negativa, como (30), la segunda oración expresa un evento posterior al de la principal; mientras

11 Cano Aguilar (2011: 484-485) encuentra en su corpus que la mayoría de las finales con no sea que aparecen en diálogos, pero también descubre algunas que reproducen un discurso de manera indirecta: Por eso era mejor que comenzasen a leer Código o Digesto o materias grandes, por que oyentes antiguos les conociesen las faltas y les avisasen dellas si algún error o necedad dijesen, no se las creyesen como las creen los institutarios (Juan de Arce de Otálora, Coloquios de Palatino y Pinciano, II, 1163). En estos ejemplos las subordinadas (con el verbo en imperfecto de subjuntivo) siguen siendo prospectivas, aunque lo sean con respecto a otro pasado.

12 Galán Rodríguez (1999: 3621) define la finalidad por oposición a la causalidad de la siguiente manera: «Las oraciones finales manifiestan una relación de causa-efecto en la que el efecto se interpreta como posterior (propósito virtual), frente a la anterioridad (motivo realizado) que designan las causales». Para un estudio de las finales y las causales desde una perspectiva tipológica, cf. Cristofaro (2005a, 2005b). 
que en los de interpretación causal, como (31), la relación entre los dos eventos es de anterioridad:

(30) — $¡$ Ah, señor don Quijote, mire por amor de Dios lo que hace! ¡No tengamos que llorar para toda nuestra vida! (DQA. XXII).

(31) Tome vuestra merced, señor licenciado; rocíe este aposento, no esté aquí algún encantador de los muchos que tienen estos libros, y nos encanten, en pena de las que les queremos dar echándolos del mundo. (DQ. I 6, 60-61).

Por otra parte, parece que existe cierta relación entre qué interpretación se le da a la construcción y el control del predicado de la subordinada. Con «control» nos referimos, como ya señalamos anteriormente, a una propiedad de un agente volitivo que implica capacidad para desencadenar la acción:

En general resultan absurdos en esta construcción los predicados que designan propiedades, acciones involuntarias y procesos experimentados (sentir alguien frío para que..., medir alguien casi dos metros para que...), ya que no aportan ningún agente al que se pueda atribuir la acción consciente o voluntaria exigida por el concepto mismo de 'finalidad'. (RAE-ASALE 2009: 46.7c)

Como veremos en el apartado siguiente, la interpretación causal o final de la subordinada depende fundamentalmente de que el cumplimiento del evento del acto de habla previo se conceptualice como un remedio para que no se cumpla el evento de la subordinada, o bien como un remedio de las consecuencias del cumplimiento de ese evento. Esa diferencia en la conceptualización se puede traducir, como hemos visto ya, en una diferencia temporal, pero también en una diferencia de control del sujeto sobre el predicado de la subordinada.

Así, los predicados menos controlados (entre ellos los inacusativos) suelen darse cuando el evento de la subordinada se conceptualiza como incontrolable, de manera que el acto de habla previo significa una manera de resistirse a ese evento, de paliar sus consecuencias:

(32) Trae el paraguas, no sea que llueva esta tarde.

(33) Habla más bajo, no sea que haya alguien conocido.

(34) Voy a dejar todo preparado, no sea que nazca el niño esta noche.

(35) Voy a terminar esto de una vez, no sea que me muera mañana.

Sin embargo, estos ejemplos revelan únicamente una tendencia, pues el hablante también puede conceptualizar como controlable un evento cuyo predicado está poco controlado: 
(36) Come bien, no sea que te desmayes en clase de kung fu.

Hay ejemplos incluso en los que caben las dos interpretaciones, causal o final negativa. Es el contexto lo que desambigua la interpretación:

(37) Haz la tortilla más grande, no sea que luego falte.

(38) Vámonos ya, no sea que te arrepientas.

(39) Llévate un libro, no sea que te aburras.

Otra diferencia fundamental entre las construcciones con interpretación final y las de interpretación causal es la distinta naturaleza que tiene la negación en cada una de ellas ${ }^{13}$ :

(40) Trae el paraguas, no sea que se mojen los libros.

'Te ordeno que traigas el paraguas para que NO exista la posibilidad de que se mojen los libros / para que exista la posibilidad de que NO se mojen los libros'

(41) Trae el paraguas, no sea que llueva esta tarde.

'Te ordeno que traigas el paraguas, porque existe la posibilidad de que llueva esta tarde (para que NO exista la posibilidad de que se cumplan las consecuencias de que llueva esta tarde)'

En la construcción desiderativa previa a la construccionalización, el ámbito de la negación era el predicado de la subordinada dependiente del verbo de suceso sea: el evento significado era algo no deseable, "mojarse los libros" o "llover esta tarde".

Cuando se construccionaliza, la negación en la subordinada de interpretación final no solamente señala ya que el evento "mojarse los libros" es no deseable, sino que, fundamentalmente, "no mojarse los libros" se reanaliza como el efecto que previsiblemente tiene el cumplimiento del evento significado por la oración principal.

En cambio, en la subordinada de interpretación causal, "no llover esta tarde" no se reanaliza ni como el efecto ni como la causa del cumplimiento del evento significado por la oración principal. El evento sobre el que tiene ámbito la negación no es ese, sino un evento que no tiene por qué explicitarse, que significa las consecuencias derivadas de "llover esta tarde": "no cumplirse las consecuencias de llover esta tarde" es lo que se reanaliza, cuando la subordinada tiene interpretación causal, como el efecto que previsiblemente tiene el cumplimiento del evento significado por la oración principal.

13 Para un estudio detallado de la negación en español, véanse Bosque (1980) y Sánchez López (1999). 
De hecho, es muy corriente que ese evento se explicite coordinándose a la oración de interpretación causal:

(42) Trae el paraguas, no sea que llueva esta tarde y se mojen los libros ${ }^{14}$.

'Te ordeno que traigas el paraguas, porque existe la posibilidad de que llueva esta tarde, para que NO exista la posibilidad de que, en consecuencia, se mojen los libros'

En relación con lo anterior está la siguiente propiedad sintáctica, que distingue a la construcción con interpretación final negativa de la de interpretación causal: solamente en la primera es posible explicitar una causa dentro de la segunda oración (43), que retoma mediante deíxis, en realidad, la causa que es el acto de habla anterior mismo:

(43) Trae el paraguas, no sea que se mojen los libros por no cogerlo.

(44) Trae el paraguas, no sea que llueva esta tarde * por no cogerlo.

Lógicamente, cuando la subordinada tiene interpretación causal, no se puede retomar una causa (44), porque es la propia oración la causa del acto de habla anterior. Solamente se puede explicitar una causa cuando se explicita el evento que significa las consecuencias derivadas del cumplimiento del evento de la subordinada, como veíamos en (42). Pero entonces la causa explicitada es un complemento de la oración coordinada con la subordinada causal:

(45) Trae el paraguas, no sea que llueva esta tarde y se mojen los libros por no cogerlo.

\subsection{Propiedades pragmáticas: un acto de habla dependiente de otro}

En el apartado 3.1. hemos visto cómo la oración de no sea que se interpreta en el nivel del discurso.

Lapesa (1978) distingue dos niveles para las causales del español, oracional y del discurso: uno en que la causal es causa de la oración (El suelo está mojado porque

$14 \quad$ Un ejemplo de este tipo del Quijote (DQ. I 6, 60-61): «Tome vuestra merced, señor licenciado; rocíe este aposento, no esté aquí algún encantador de los muchos que tienen estos libros, y nos encanten, en pena de las que les queremos dar echándolos del mundo».

15 Los siguientes ejemplos son del CNDHE:

(a) Ola, no reçongues, no sea que por tu reçongar se hierre esta obra (Ruiz de Alarcón 1629, Tratado de las supersticiones y costumbres gentílicas...).

(b) Demos con rendimiento / fin al sainete, / no sea que por largo / también se yerre (Lanini Sagredo c1670, «Baile del herrador»). 
ha llovido) y otro en el que justifica un acto de habla (Ha llovido, porque el suelo está mojado) ${ }^{16}$.

La existencia de distintos niveles puede defenderse, además de por las diferencias de comportamiento sintáctico de los elementos gramaticales que operan en ellos, porque hay lenguas que tienen especializadas diferentes conjunciones para distintos niveles, por ejemplo, el francés (Sweetser 1990: 82): «parce que "because" is used specifically for content conjunction, while puisque is the correct causal conjunction at the epistemic or speech-act level».

Sweetser (1990: 76-77), en cambio, separa en tres niveles las causales según qué tipo de conjunción tengan:

(a) John came back because he loved her. (content conjunction)

(b) John loved her, because he came back. (epistemic conjunction)

(c) What are you doing tonight, because there's a good movie on. (speech-act conjunction)

A diferencia de Lapesa (1978) (y de Ross 1970 y Davison 1973), Sweetser (1990) subdivide las causales del nivel discursivo en dos categorías: una que expresa un conocimiento previo del que se deduce una conclusión (b) y otra que justifica un acto de habla (c).

Baños (2014: 31) distingue igualmente dos tipos de causales del nivel del discurso y ofrece los siguientes ejemplos de cada una de ellas:

(46) Tu madre no está, porque / pues la he llamado y no coge el teléfono.

(47) Ya que has sacado el tema, ¿me vas a invitar a la boda?

Del primer ejemplo dice que «el hablante señala la fuente o evidencia que justifica la validez del contenido proposicional, es decir, el valor de verdad de la oración principal»; mientras que del segundo ejemplo dice que en él «la oración causal justifica el propio acto de habla, (...) expresa la razón por la que se enuncia la propia oración interrogativa». Las del primer tipo se interpretan en el nivel de la proposición y las del segundo tipo, en el nivel de la ilocución. Esta distinción se puede aplicar igualmente a las finales.

Las oraciones de no sea que funcionan en el nivel de la ilocución: por ello se pueden parafrasear por Lo digo / pregunto / ordeno porque / para que... (Baños 2014: 35).

En RAE y ASALE $§ 46.7 j$ se apunta tímidamente algo de esto:

$\overline{16}$ Bello, en 1847, y Meyer Lübke (para el latín) en 1899, ya se referían a esa oposición. 
Estas construcciones se asimilan en ciertos casos a finales que corresponden al plano de la enunciación $(\$ 46.5 \mathrm{~g})$. Una posible paráfrasis del ejemplo de Montenegro citado en el $\$ 46.7 i$ (Ten cuidado no te pase a ti algo parecido) podría ser, por consiguiente, Te pido que tengas cuidado para que no te pase a ti algo parecido.

Aunque en 46.5g no se distinguen, como en Sweetser (1990) o en Baños (2014), dos tipos de oraciones en el nivel del discurso, todos los ejemplos que se aducen son del mismo tipo, del nivel de la ilocución, como el siguiente:

(48) Mira, es más pequeña que yo, para que veas, ¿eh? (Sánchez Ferlosio, Jarama).

Así pues, la construcción que analizamos está formada por dos actos de habla, uno principal, que aparece siempre en primer lugar, y uno subsidiario, que justifica la enunciación del acto de habla previo.

Este análisis se puede reflejar muy bien en el modelo de la Gramática Funcional del Discurso: la oración de interpretación causal o final emerge en el nivel interaccional (pragmático) como un acto de habla dependiente, que desempeña la función Motivación (Hengeveld y Mackenzie 2008: 53-54):

Dependence holds between Discourse Acts to which the Speaker gives unequal communicative status. Dependence is shown in underlying representation through the presence of a rhetorical function on the Subsidiary Discourse Act. A Subsidiary Discourse Act may have various rhetorical functions, such as Motivation, Concession, Orientation, and Correction. Consider a Move such as (13):

(13) Watch out, because there will be trick questions in the exam.

Here, the Speaker's strategy is oriented to warning the Addressee. This strategy is implemented by uttering two (intonationally distinct) Discourse Acts in succession, one with an Imperative Illocution and one with a Declarative Illocution (...). The presence of the conjunction because indicates that the second Discourse Act is intended to be understood as subsidiary to the first, specifically as indicating the Speaker's Motivation for uttering the Imperative Illocution.

El hablante quiere ofrecer una justificación del acto de habla anterior, en muchos casos porque se trata de un acto directivo, que supone una amenaza para la imagen de su interlocutor. El acto de habla introducido por la oración de no sea que atenúa entonces el acto de habla principal, que no se interpreta ya como una orden, sino como un consejo o una recomendación ${ }^{17}$. Sin embargo, aunque sea lo más frecuente, no solo se combinan con actos de habla directivos, como vimos en 3.1.

17 Esta misma función la cumplen las causales que estudia Iglesias Recuero (2000), cuando se combinan con la modalidad imperativa en la principal, del tipo Deja eso, que te vas a hacer daño o No hagas tanto ruido, que estoy intentando concentrarme. 
Justificar el acto de habla anterior es una función corriente de las causales en las lenguas del mundo (aunque ya hemos visto cómo las finales también pueden desempeñar esa función) y parece que el orden tipológicamente no marcado es aquel en el que la oración principal precede a la causal (cf. Diessel y Hetterle 2011). Las finales pueden anteponerse quizá con mayor facilidad, pero el orden tipológicamente no marcado es el mismo. Por esta razón no es extraño que el orden en que se ha fijado la construcción sea el de 1) acto de habla principal, 2) acto de habla subsidiario.

Por otro lado, la función pragmática Motivación puede darse de dos maneras en la construcción, que se reflejan precisamente en la interpretación causal, o bien final negativa, de la subordinada:

a) cuando el hablante conceptualiza el acto de habla previo como un remedio, el cumplimiento del evento de la segunda oración es un temor que puede evitarse, es algo controlable, remediable: entonces la interpretación es final negativa;

b) en cambio, cuando conceptualiza el acto de habla previo como una forma de resistirse a un evento que es incontrolable, irremediable, lo único que se puede remediar son las consecuencias derivadas de ese evento incontrolable: la interpretación de la subordinada es entonces causal.

En cualquier caso, el evento significado por la oración de no sea que es algo que el hablante desea evitar, o desea evitar sus consecuencias, no desea su cumplimiento, y por ello busca un remedio o una forma de resistirse a ello.

La oposición controlable / incontrolable es una oposición pragmática, que se conceptualiza en la mente del hablante, y se traduce en la gramática con diferentes recursos, como ya hemos visto: uno de ellos, la relación temporal entre el evento significado por la subordinada y el evento de la principal; otro, la diferente interpretación de la negación; y a veces, el control del sujeto sobre el predicado de la subordinada.

La condición que cumplen los eventos incontrolables no es simplemente que el sujeto no tenga capacidad para desencadenar la acción. De hecho, en ejemplos como No escribas en la pizarra, no sea que venga el profesor antes de tiempo esto es muy discutible. En este caso no es relevante si el profesor tiene o no control sobre la acción. Lo que desencadena la interpretación causal de la segunda oración, y que el evento significado en ella se entienda como incontrolable, es que el hablante y el oyente no pueden evitar, no tienen capacidad de evitar ese evento. Se trata por ello de una condición pragmática, estrechamente ligada a la situación en la que se enuncia la frase ${ }^{18}$.

18 De ahí las etiquetas de «precautorio» o «preventivo» que se le han asignado a la construcción en la bibliografía (Cano Aguilar 2011; RAE-ASALE 2009; Montolío 1999; Gómez Torrego 1988), 
En este sentido también Núñez (1998: 602) se refiere al "control" del hablante (capacidad para evitar el evento) cuando estudia lo que él denomina las «construcciones condicionales procausales» en latín ${ }^{19}$, equivalentes a la paráfrasis (también posible en las oraciones de interpretación causal que aquí estudiamos) con por si (acaso):

la diferencia entre los enunciados finales y estos condicionales estaría en que los primeros se presentan como "no problemáticos", en la medida en que implican la idea de alcanzar la realización de la predicación contenida en la oración subordinada, mientras que las introducidas por $\varepsilon \dot{i}$ / si carecen de esa implicación y expresan "cierta incertidumbre" sobre la realización de la finalidad. En muchos casos, la realización de lo deseado no está bajo el control de la persona que lo expresa (normalmente el sujeto de la predicación principal) sino que depende de algún otro (...). De ahí que uno de los rasgos característicos de esas construcciones sea la gran frecuencia de su uso con verbos de voluntad, que explícitamente muestran que la realización depende del deseo de alguien, y posibilidad, que enfatizan que la realización del predicado subordinado es incierto.

\section{LA CONSTRUCCIONALIZACIÓN DE UNA DESIDERATIVA NEGADA}

En este apartado se examinará fundamentalmente la relación que hay entre la construcción que estamos estudiando y la modalidad desiderativa.

La segunda oración de nuestra construcción lleva el verbo en subjuntivo, que es una manera de reflejar la modalidad irrealis en español, modalidad propia de las desiderativas (entre otras construcciones):

Moods are verbal inflections reflecting modality. They may directly encode grammatical differences related to speech acts, as in the subjunctive form tenga in ¡Tenga un buen

aunque no se expliquen nunca claramente ni se distinga bien con ellas lo que es contenido semántico de lo que es contenido pragmático. En RAE-ASALE (2009: §24.1m) se dice de la segunda oración que «introduce el motivo de cierta prevención manifestada en el contexto previo». Gómez Torrego (1988: 74) habla de «un valor entre final y precautorio». Montolío (1999: 3678) se refiere a la misma construcción con una etiqueta semejante, la de «preventivo». Esta asignación se debe, según Cano Aguilar (2011: 485-486), a que la segunda oración significa una circunstancia que trata de evitarse, pero aplica su definición no solo a ejemplos del tipo Trae el paraguas, no sea que se mojen los libros, sino también a los que él denomina de «finalidad secundaria», del tipo Trae el paraguas, no sea que llueva esta tarde. En los ejemplos de ese tipo parece claro que el evento expresado por la segunda oración no trata de evitarse, porque, de hecho, que suceda o no es algo incontrolable («que llueva esta tarde», en el ejemplo).

19 Construcciones como ibo hinc intro, perscrutabor fanum, si inueniam uspiam aurum. (Plaut. Aul.620). 
dia! 'Have a nice day!,' as opposed to the indicative tiene in Tiene un buen dia ' $\mathrm{S} / \mathrm{he}$ is having a nice day.' (Bosque 2012: $373^{20}$ )

El tipo de construcción que estamos aquí estudiando lo hemos documentado también en latín ${ }^{21}$, aunque no tenemos pruebas de que haya una relación genética entre ambas construcciones. En latín se yuxtaponen también dos oraciones, una de ellas encabezada por ne (forte), unas veces con interpretación causal (49) y otras, con final negativa (50), que funciona también en el nivel pragmático como una Motivación, una justificación del otro acto de habla:

(49) certusque aliquod moliri flagitium dedi quidem potionem, dedi; sed futurae quaestioni praecauens non statim pretium, quod offerebatur, accepi, sed "ne forte aliquis", inquam, "istorum, quos offers, aureorum nequam uel adulter repperiatur, in hoc ipso sacculo conditos eos anulo tuo praenota, donec altera die nummulario praesente comprobentur." («y como estaba seguro de que tramaba alguna fechoría, le di, es cierto, el brebaje, se lo di, pero previendo que hubiera una investigación posterior, no le acepté en ese momento el precio que me ofrecía, sino que le dije:

- No vaya a ser que alguna de las monedas de oro que me entregas resulte falsa o defectuosa: márcalas, por tanto, con tu anillo sin sacarlas de esta misma bolsa hasta que mañana hagamos venir a un cambista para comprobarlo», Apul.Met.10.9.9-14).

(50) praecipue gelidum stipula cum fronde caduca / sterne solum, ne forte rigor penetrabile corpus / urat et interno uastet pecuaria morbo.

(«Sobre todo tapiza el suelo helado con paja y hojas secas, no sea, tal vez, que el rigor del frío queme los cuerpos indefensos y una plaga arruine internamente al ganado.», Calp.Ecl.5.116-118).

Fruyt (2011: 716-718) y Lakey (2015), que estudian el proceso de gramaticalización que se produce en las oraciones independientes con ne en general al reanalizarse como subordinadas, consideran que este comienza en ejemplos en los que se reproduce en estilo indirecto una orden negada o una prohibición, como:

(51) Miles, nunc adeo edico tibi / ne uim facias ullam in illam.

(«Ahora, soldado, te doy una orden: que no le hagas la menor violencia», Ter. Eun.806-807).

Esta hipótesis no es aceptable para el tipo de construcción que aquí se analiza, ya que se documentan en las mismas épocas que estudian los autores ejemplos de

20 Sobre la oposición indicativo / subjuntivo en español, véase también Bosque (1990).

21 En italiano se documenta Portati l'ombrello, non sia mai (che) si metta a piovere. 
oraciones con $n e+$ subjuntivo combinados con modalidades distintas a la enunciativa en la principal, y que, por tanto, no pueden proceder de la reproducción de un discurso indirecto que se reanaliza como subordinado a un verbo de lengua; por otra parte, la oración de ne (forte) no es nunca una orden o una prohibición, sino en todo caso una justificación para haber dado una orden o hecho una prohibición (entre otros posibles actos de habla).

Lakey (2015) se refiere también a un tipo de condicionales poco prototípicas (que podrían clasificarse también como causales potenciales o hipotéticas) que hace derivar igualmente de las imperativas negadas:

(52) ego ibo ad fratem ad alios captiuos meos, / uisam ne nocte hac quippiam turbauerint. ( «Yo voy a casa de mi hermano a dar una vuelta a los otros cautivos, a ver si han hecho esta noche alguna de las suyas», Plaut.Capt.126-127).

Sin embargo, como enseguida veremos, tanto en esta última construcción del latín como en la del español que estamos aquí analizando, lo que se ha construccionalizado es una desiderativa, no una imperativa.

Por otra parte, el adverbio forte, que puede aparecer en la construcción latina, sería, según Rodríguez Molina (2013: 110-111), un adverbio «de voluntad no intencional», es decir, que especifica «que un evento ha tenido lugar de manera fortuita o casual, sin la instigación de un agente o, a menudo, significan que el evento ha sucedido fuera del control de un agente, en caso de que esté previsto en la estructura argumental del verbo».

En español moderno no aparece un adverbio equivalente en la construcción. No obstante, ese adverbio forte se vería sustituido en castellano antiguo por otras locuciones adverbiales, entre ellas por ventura. Rodríguez Molina (2013: 114-119) estudia los usos de esta locución en la historia del español y concluye que «Por ventura es la locución no intencional más empleada a lo largo de toda la Edad Media hasta el siglo XVI (...) y, aunque todavía se emplea en el XVII (...), su uso productivo como locución adverbial no intencional no llega vivo al siglo XVIII».

Sin embargo, Rodríguez Molina no recoge entre las construcciones de por ventura ninguna que signifique lo mismo que las construcciones latinas que acabamos de ver con ne forte, y que las modernas Trae el paraguas, no sea que \{llueva esta tarde / se mojen los libros\}. A continuación, ofrecemos unos ejemplos de por ventura con este uso, pertenecientes al siglo XIII' ${ }^{22}$. La segunda oración aparece introducida

22 Está todavía por hacer un análisis exhaustivo de la variación de la construcción en la historia del español. El artículo citado de Cano Aguilar trae información sobre ella en el siglo XVI. También 
por que y puede tener interpretación causal (53) o final negativa (54). Pertenecen a la Estoria de Alexandre, contenida en la cuarta parte de la General Estoria. Son especialmente interesantes porque contamos con una reconstrucción del texto latino que estaban traduciendo:

(53) E ante que moviesse d'allí Alexandre, rogó 1 mucho aquel so princep Eumilo e díxo $\cdot 1$ : «Muy grand emperador, pído-te por merced que me dexes passar contigo este río e yr d'allí adelant otrossí contigo, que por ventura que te non acaesca ý alguna cosa, e como vas señero que non ayas con qui la partir e la conortar». / Eumilo autem rogabat eum dicens: «Maxime imperator, permitte me transire tecum ipsum fluuium et uenire, ne forte eueniat tibi aliqua angustia» (EA. XXII.41-46 / 60.28-30).

(54) Enviámos-vos esta nuestra carta e significámos-vos que, ante que a nuestras tierras vengades, ante cuededes cómo vernedes que por ventura non suffrades de nos angostura e desondra, lo que de ningunos non soffristes fasta agora. / Scribimus et significamus uobis ut, antequam ueniatis in finibus nostris, cogitetis quomodo ueniatis, ne forte patiasis a nobis turpitudinem et angustiam quam a nullo passus est. (EA. L.5-9 / 83.5-8).

En los apartados anteriores hemos señalado que la función pragmática Motivación se codifica mediante oraciones de interpretación causal y final, que se caracterizan por ciertas propiedades que comparten con las construcciones desiderativas: esta relación no es casual, sino que se debe a que estas últimas están en el origen de esta construcción.

Como ya señalamos anteriormente y conviene recordar aquí, las construcciones desiderativas se caracterizan por que ni el hablante ni el destinatario tienen control sobre el cumplimiento del evento significado en ellas, mientras que en las construcciones imperativas el destinatario sí tiene control sobre el evento. Los dos tipos de construcción pertenecen a la modalidad irrealis, que señala que el contenido no es real en el mundo en el que se habla.

La desiderativa que se construccionaliza es una desiderativa negativa sin una conjunción o adverbio introductorio y sin otra marca de modalidad más que el subjuntivo, que es, además, marca de irrealis. El verbo de la desiderativa es un verbo

se citan ejemplos de la construcción en la Edad Media en Carrera de la Red (1989: 44) (de Berceo, concretamente) bajo la etiqueta «oraciones yuxtapuestas con matiz consecutivo-final»: por ejemplo, tuelle d'est'monesterio esta tribulación, / nón caya la tu casa en tan grand perdición (S. Domingo, 195 d). En Sánchez Jiménez (2002: 308) también hay un ejemplo: Donna Llambla callad non uos pese soffrit uos (EST II, 86r. 17-18). En ninguno de estos dos últimos artículos se ofrece un análisis de la construcción. 
de suceso: ser en la desiderativa que da origen a la variante de la construcción que estamos estudiando, pero en otras variantes que pueden documentarse en español moderno es suceder, pasar o ir a ser. El verbo de suceso introduce una subordinada completiva en función de sujeto.

Gómez Torrego (1988) considera no vaya a ser que y no fuera a ser que locuciones conjuntivas (y también no sea que y las demás variantes de la construcción). Sin embargo, que existan estas variantes, restringidas, por un lado, a los verbos de suceso, y, por otro lado, que sirvan para la expresión de distintos tiempos, es un indicio de que el verbo de suceso, en presente o en imperfecto de subjuntivo, no se ha lexicalizado, sino que, junto a la negación, la subordinada completiva que introduce y junto a la oración principal (que precede a toda la oración de no sea que), constituye una construcción.

En los siguientes fragmentos de los siglos XVI y XVII, tomados del CNDHE, puede apreciarse cómo la oración de no sea que tiene una naturaleza distinta a la que tiene en los ejemplos que hemos visto hasta ahora de la construcción:

(55) ¿Qué necesidades, qué guerras en tierra y mar, qué sucesos tan infelices, qué desconsuelos particulares y públicos que parece que han jurado contra nosotros los elementos, el cielo, y cuánto poder bastan a mostrar sus estrellas? ¡Oh, no sea que el autor de ellas arme el orbe de la tierra contra los entendidos, como amenazó la Sabiduría a los insensatos! (Paravicino 1633, Jesucristo desagraviado).

(56) Ahora no los matan y apedrean, sino los honran y regalan. A Dios gracias, que nos ha traído á mejor tiempo. Pero no sea que los diligencieros nos concertamos con los arrendadores, y ó por temor de nuestro daño ó codicia del provecho, no les apretamos ni hacemos instancia (Cabrera a1598, De las consideraciones sobre todos los evangelios de la Cuaresma).

En (55) el verbo de la subordinada completiva (arme) está también en subjuntivo (concordancia temporal esperable, por otra parte), pero toda la oración de no sea que no es la causa ni el efecto de la interrogativa que la precede; se le antepone, además, la interjección oh. En (56), en cambio, se le antepone la conjunción pero; la oración tampoco es la causa ni el efecto de A Dios gracias, que nos ha traído á mejor tiempo. En este ejemplo, aparece, además, indicativo: concertamos. Esta falta de concordancia con sea es algo fuera de lo común, o al menos solo hemos encontrado otro ejemplo semejante en el CNDHE, (57), en el que la oración de no sea que lleva un verbo en indicativo (huyeron) en la subordinada. En este caso es a su vez argumento (CD) de la desiderativa plegue a su Majestad divina: 
(57) Misterio es, que sólo Dios lo sabe; y plegue a su Majestad divina no sea que entonces huyeron los demonios por aquel tiempo que fue de grande conversión de ánimas para Dios y de edificación, y que después hayan vuelto por haberles dado lugar los cristianos para que se enseñorearan de nuevo con abusos y malos ejemplos, y ofensas de Dios nuestro Señor, y escándalos de los pequeñuelos (Mendieta a1604, Historia eclesiástica indiana).

En cualquier caso, las características señaladas en los ejemplos anteriores son indicios de que la oración es independiente en esos contextos, no forma parte de una construcción mayor. Nada de esto era posible, en cambio, en los ejemplos que veníamos analizando.

La oración encabezada por no sea que es una desiderativa en (55-56), ya que el hablante expresa el deseo de que se cumpla el evento significado en ella y la impotencia, la falta de control, para hacerlo él mismo o para que lo haga su interlocutor. La construcción desiderativa no está encabezada por un adverbio o una conjunción, que es una manera corriente de hacer desiderativas potenciales en esa época (tanto la desiderativa que comienza por no sea que, como la subordinada de interpretación causal o final negativa en la construcción, se refieren a estados de cosas posibles, nunca imposibles). La negación excluye el cumplimiento de un evento, lo que se desea es que precisamente ese evento no se cumpla, mientras que en un ejemplo de desiderativa sin negación, como (58), lo que se desea es que se cumpla el evento enunciado:

(58) Encomendados sean a Satanás y a Barrabás tales libros, que así han echado a perder el más delicado entendimiento que había en toda la Mancha ( $D Q$. I 5).

Pero en los siglos XVI y XVII también se documentan otro tipo de ejemplos como el siguiente:

(59) Señor -dixo el príncipe Arsileo-, al esforçado príncipe don Belianís hallaréys aquí en esta arboleda, en saliendo del castillo, vos le llamad pues que auéys hablado al gigante, no sea que desconociéndome por las armas, se pierda todo nuestro hecho (Jerónimo Fernández 1547, Belianis de Grecia, apud Cano Aguilar 2011: 487).

En este caso la oración de no sea que lleva un subjuntivo en la subordinada en función de sujeto, es dependiente de la anterior oración (vos le llamad) y guarda una relación de causa-efecto con ella. Es otro caso del tipo Trae el paraguas, no sea que se mojen los libros, como lo era (30).

Se puede apreciar, a la vista de este ejemplo y los anteriores, que en los siglos XVI y XVII era productiva todavía la construcción desiderativa que se reanalizó como 
Motivación en la nueva construcción y convivía con esta última, pues se documentan en los textos de esa época ejemplos no solo de oraciones finales negativas y causales en el nivel de la ilocución, sino también ejemplos en los que todavía la oración de no sea que es una construcción desiderativa independiente. En español moderno, en cambio, no hemos documentado construcciones desiderativas independientes encabezadas por no sea que, ni con subjuntivo ni con indicativo en la subordinada: ?? No sea que llueve esta tarde!; ?? No sea que llueva esta tarde!.

Cano Aguilar (2011: 487) defiende que es precisamente a finales del siglo XVI y durante el XVII cuando las oraciones de no sea que alcanzan su apogeo y sitúa a finales del XIX el origen de la variante encabezada por no vaya a ser que.

En la construcción que aquí analizamos ya vimos cómo la segunda oración, independientemente de su interpretación causal o final negativa, se caracteriza también por su virtualidad o irrealidad, como lo hacen, por otra parte, las desiderativas: cuando el hablante las pronuncia, está en un mundo que no es el mismo que aquel que considera deseable.

\section{CONCLUSIONES}

Las oraciones yuxtapuestas Trae el paraguas, no sea que llueva esta tarde y Trae el paraguas, no sea que se mojen los libros son ejemplo de un tipo de construcción, ya que conforman una unidad con unas características sintácticas y semánticas determinadas; tienen unas propiedades discursivas específicas y la función pragmática que desempeña la segunda oración en ella es siempre la misma, Motivación: el hablante ofrece una justificación del acto de habla anterior, en la mayoría de los casos porque se trata de un acto directivo, que supone, por tanto, una amenaza para la imagen de su interlocutor.

La segunda oración está encabezada por una negación y el verbo es un verbo de suceso (ser), que en otras variantes que se documentan de la construcción es suceder, pasar o ir a ser. Este verbo de suceso introduce una subordinada completiva en función de sujeto.

La construcción está formada por dos actos de habla: uno principal, que aparece siempre en primer lugar, y uno subsidiario, que justifica la enunciación del acto de habla previo, y en la mayoría de los casos, lo atenúa.

El hablante no desea el cumplimiento del evento introducido por la oración de no sea que y por ello busca un remedio o, si no tiene remedio, una forma de resistirse a ello. Así, la función pragmática Motivación del acto subsidiario se codifica en el discurso, respectivamente, mediante una oración de interpretación final negativa, o bien mediante una oración de interpretación causal: 
1) cuando el hablante conceptualiza el acto de habla previo como un remedio, el evento de la segunda oración es un temor que puede evitarse, es algo controlable: entonces emplea una oración final negativa;

2) en cambio, cuando conceptualiza el acto de habla previo como una forma de resistirse a un evento que es incontrolable, este segundo evento lo expresa mediante una oración causal (potencial o hipotética).

La función pragmática determina, por un lado, la codificación morfosintáctica de la construcción:

(a) Las dos oraciones que conforman la construcción están yuxtapuestas y la segunda mantiene con la primera una relación de dependencia o subordinación.

(b) La segunda oración puede tener interpretación final negativa (Trae el paraguas, no sea que se mojen los libros) o causal (Trae el paraguas, no sea que llueva esta tarde) y aparece siempre pospuesta a la otra oración, el orden tipológicamente no marcado de las finales y las causales.

(c) Esta segunda oración se combina con otra oración de cualquier modalidad, independientemente de que su interpretación sea final negativa o causal, aunque mayoritariamente con la modalidad imperativa.

(d) La posibilidad que tiene la oración de no sea que de combinarse con una oración previa de distintas modalidades y, por otro lado, la entonación de coma de esta última o la pausa entre ambas oraciones refleja el funcionamiento de la segunda (la de no sea que) en el nivel del discurso.

La función pragmática también determina la interpretación de la construcción. Que la interpretación de la subordinada sea causal o final negativa depende fundamentalmente de que el cumplimiento del evento del acto de habla previo se conceptualice como un remedio para que no se cumpla el evento de la subordinada, o bien como un remedio de las consecuencias del cumplimiento de ese evento.

Esa diferencia en la conceptualización se traduce en una diferencia temporal, en la interpretación de la negación, pero también puede traducirse en una diferencia de control del sujeto sobre el predicado de la subordinada:

(a) Las oraciones de interpretación causal pueden ser prospectivas o no, pero las finales negativas solo pueden ser prospectivas: cuando la segunda oración expresa tiempo presente o antepresente, la interpretación es necesariamente causal. 
(b) La negación en la subordinada de interpretación final afecta al evento de la subordinada: que ese evento no se cumpla es el efecto que previsiblemente tiene el cumplimiento del evento significado por la oración principal. Mientras que, en la subordinada de interpretación causal, el evento sobre el que tiene ámbito la negación no es el de la subordinada, sino un evento que no tiene por qué explicitarse, que significa las consecuencias derivadas del cumplimiento del evento de la subordinada. Es decir, "no cumplirse las consecuencias del evento de la subordinada" es el efecto que previsiblemente tiene el cumplimiento del evento significado por la oración principal. Este evento, de hecho, a veces se explicita coordinándose otra oración a la oración subordinada de interpretación causal: Trae el paraguas, no sea que llueva esta tarde y se mojen los libros.

(c) Los predicados menos controlados (como, por ejemplo, los inacusativos) aparecen normalmente cuando el evento de la subordinada se conceptualiza como incontrolable, cuando el acto de habla previo significa una manera de resistirse a ese evento, de paliar sus consecuencias. Se trata, en este caso, de una tendencia, no de una propiedad necesaria ni suficiente de las oraciones de interpretación causal.

Que tanto las oraciones de interpretación final negativa como las de interpretación causal puedan ser prospectivas, la concepción del evento de la subordinada como no deseable en ambos casos, o la pertenencia de ese evento a la modalidad irrealis, son consecuencia de que la oración que se construccionalizó como subordinada sea una desiderativa negativa.

En los siglos XVI y XVII todavía se documentan no solo oraciones de no sea que de interpretación final negativa y de interpretación causal en el nivel de la ilocución, sino también ejemplos en los que todavía la oración de no sea que es una construcción desiderativa independiente. Podemos distinguir claramente si la segunda oración todavía funciona de manera independiente por una serie de indicios:

(a) Si la oración no guarda una relación de causa-efecto o efecto-causa con la oración anterior.

(b) Si se intercalan entre la oración de no sea que y la anterior conjunciones que no son causales o finales, como pero.

(c) Si se anteponen interjecciones como $o h$ a la oración encabezada por no sea que. 
Este mismo tipo de construcción lo hemos documentado también en latín, encabezada la subordinada de interpretación final negativa o de interpretación causal por ne (forte).

\section{CORPUS}

[Apuleyo] Martos, J. (2003): Las metamorfosis o El asno de oro, II. Madrid: Consejo Superior de Investigaciones Científicas.

[Avellaneda] Gómez Canseco, L. (2000): El ingenioso hidalgo don Quijote de la Mancha. Madrid: Biblioteca Nueva.

[Calpurnio] Korzeniewski, D. (1971): Bucolica aetatis neronianae. Darmstadt: Wissenschaftliche Buchgesellschaft.

[Calpurnio] Correa Rodríguez, J. A. (1984): Poesía latina pastoril de caza y pesca. Madrid: Gredos.

[Cervantes] Rico, F. (2005): Don Quijote de la Mancha. Junta de Comunidades de Castilla-La Mancha.

[CNDHE] Instituto de Investigación Rafael Lapesa de la Real Academia Española (2013): Corpus del Nuevo diccionario histórico (CDH) [en línea]. <http://web. frl.es/CNDHE>

[Estoria de Alexandre] González Rolán, T. y Saquero Suárez-Somonte, P. (1982): La historia novelada de Alejandro Magno. Universidad Complutense de Madrid.

[Plauto] Lindsay, W. M. (1968): T. Macci Plauti comoediae. Tomus I. Oxford: E Typographeo Clarendoniano.

[Plauto] González-Haba, M. (1992): Comedias I. Madrid: Gredos.

[Terencio] Rubio, L. (1991): Comedias. Volumen I: La andriana; El eunuco. Madrid: Consejo Superior de Investigaciones Científicas.

\section{BIBLIOGRAFÍA}

BAÑos, J. M. (2014): Las oraciones causales en latín. Madrid: Escolar y Mayo.

Bello, A. (1988 [1847]): Gramática de la lengua castellana destinada al uso de los americanos. Madrid: Arco Libros.

Bosque, I. (1980): Sobre la negación. Madrid: Cátedra.

BosQue, I. (1990) (coord.): Indicativo y subjuntivo. Madrid: Taurus.

Bosque, I. (2012): "Mood. Indicative vs. Subjunctive", in J. I. Hualde, A. Olarrea, y E. O'Rourke (eds.): Handbook of Hispanic Linguistics. Oxford: Blackwell, pp. 373-394. https://doi.org/10.1002/9781118228098.ch19 
Brucart, J. M. y GAllego, Á. (2009): “L'estudi formal de la subordinació i l'estatus de les subordinades adverbials", Llengua y literatura 20, pp. 139-191.

Cano Aguilar, R. (2011): "No sea que: yuxtaposiciones en el español del s. XVI", in J. J. Bustos Tovar, R. Cano Aguilar, E. Méndez García de Paredes, A. López Serena (coords.): Sintaxis y análisis del discurso hablado en español. Homenaje a Antonio Narbona, I. Sevilla: Servicio de Publicaciones de la Universidad de Sevilla, pp. 479-494.

CARrera de la ReD, M. F. (1989): "Sobre la expresión de la finalidad en la obra de Berceo", Anuario de Estudios Filológicos 12, pp. 39-65.

Cristofaro, S. (2005a): "Purpose Clauses", in M. Haspelmath, M. Dryer, D. Gil, y B. Comrie (eds.): World Atlas of Language Structures. Oxford: Oxford University Press, pp. 506-509.

Cristofaro, S. (2005b): "Reason Clauses”, in M. Haspelmath, M. Dryer, D. Gil, y B. Comrie (eds.): World Atlas of Language Structures. Oxford: Oxford University Press, pp. 514-517.

CROFT, W. (2005): “Logical and typological arguments for Radical Construction Grammar”, in M. Fried y J. O. Östman (eds.): Construction Grammar(s): cognitive and cross-language dimensions. Amsterdam: John Benjamins, pp. 273-314. https://doi.org/10.1075/cal.3.11cro

Davison, Alice (1973): Performative verbs, adverbs and felicity conditions: an inquiry into the nature of performative verbs. Tesis Doctoral. University of Chicago: Department of Linguistics.

Diessel, H. y Hetterle, K. (2011): “Causal clauses: A cross-linguistic investigation of their structure, meaning, and use", in P. Siemund (ed.): Linguistic Universals and Language Variation. Berlín, Nueva York: Mouton de Gruyter, pp. 23-52. https://doi.org/10.1515/9783110238068.23

Fruyt, M. (2011): “Grammaticalization in Latin”, in P. Baldi y P. Cuzzolin (eds.): New Perspectives on Historical Latin Syntax 4: Complex Sentences, Grammaticalization, Typology. Berlín: Mouton de Gruyter, pp. 661-864. https://doi. org/10.1515/9783110253412.661

GAlÁN Rodríguez, G. (1992): Las oraciones finales en español. Estudio sincrónico. Cáceres: Universidad de Extremadura.

GAlÁn Rodríguez, C. (1999): “La subordinación causal y final”, in I. Bosque y V. Demonte (coords.): Gramática descriptiva de la lengua española, III. Madrid: Espasa Calpe, pp. 3597-3642. 
Gili GaYA, S. (1943): Curso superior de sintaxis española. México D.F: Minerva.

GoHL, C. (2000): "Causal relations in spoken discourse: Asyndetic constructions as a means for giving reasons", in B. Kortmann y E. Couper-Kuhlen (eds.): Cause, Condition, Concession, Contrast. Berlín, Nueva York: Mouton de Gruyter, pp. 83-110. https://doi.org/10.1515/9783110219043.1.83

Gómez Torrego, L. (1988): Perifrasis verbales: sintaxis, semántica y estilística. Madrid: Arco Libros.

Hengeveld, K. y Mackenzie, J. L. (2008): Functional Discourse Grammar: A typologically-based theory of language structure. Oxford: Oxford University Press. https://doi.org/10.1093/acprof:oso/9780199278107.001.0001

Hopper, P. y Traugott, E. C. (2003): Grammaticalization. Cambridge: Cambridge University Press. https://doi.org/10.1017/CBO9781139165525

IgLesias Recuero, S. (2000): "Gramática de la oración frente a gramática del discurso: de nuevo sobre el llamado "que" causal”, in J. J. Bustos Tovar (coord.): Lengua, discurso, texto: I simposio internacional de análisis del discurso, I. Madrid: Visor, pp. 333-344.

LaKeY, H. A. (2015): "The Grammaticalization of Latin $n \bar{e}+$ Subjunctive Constructions", Journal of Latin Linguistics 14:1, pp. 65-100. https://doi.org/10.1515/ joll-2015-0004

LANGACKER, R. W. (1987): Foundations of Cognitive Grammar: theoretical perspectives, I. Stanford: Stanford University Press.

LAPESA, R. (1978): “Sobre dos tipos de subordinación causal”, in Estudios ofrecidos a E. Alarcos Llorach, III. Oviedo: Universidad de Oviedo, pp. 173-205.

LeHMANN, C. (1985): "Grammaticalization: synchronic variation and diachronic change”, Lingua e Stile 20, pp. 303-318.

Lehmann, C. (1988): "Towards a typology of clause linkage", in J. Haiman y S. A. Thompson (eds.): Clause combining in grammar and discourse. Amsterdam: John Benjamins, pp. 181-225. https://doi.org/10.1075/tsl.18.091eh

LÓPEZ GARCÍA, Á. (1999): “Relaciones paratácticas e hipotácticas”, in I. Bosque y V. Demonte (coords.): Gramática descriptiva de la lengua española, III. Madrid: Espasa Calpe, pp. 3507-3548.

Montolío, E. (1999): "Las construcciones condicionales", in I. Bosque y V. Demonte (coords.): Gramática descriptiva de la lengua española, III. Madrid: Espasa Calpe, pp. 3643-3737. 
NARbona, A. (1990): Las subordinadas adverbiales impropias en español (II). Causales y finales, comparativas y consecutivas, condicionales y concesivas. Málaga: Ágora.

NúÑEZ, S. (1998): "Los enunciados condicionales 'procausales' en latín”, in B. García Hernández (ed.): Estudios de Lingüística latina: Actas del IX Coloquio Internacional de Lingüistica Latina. Madrid: Universidad Autónoma de Madrid, pp. 597-612.

PÉREZ Saldanya, M. (1999): "El modo en las subordinadas relativas y adverbiales", in I. Bosque y V. Demonte (coords.): Gramática descriptiva de la lengua española, II. Madrid: Espasa Calpe, pp. 3253-3322.

RAE-Asale (2009): Nueva gramática de la lengua española. Madrid: Espasa Calpe. RodríGuez Molina, J. (2013): “Trayectoria diacrónica de los adverbios de manera no intencionales", in M. P. Garcés Gómez (ed.): Los adverbios con función discursiva: procesos de formación y evolución. Madrid / Frankfurt: Iberoamericana / Vervuert, pp. 109-156.

Rojo, G. (1978): Cláusulas y oraciones. Anejo 14 de Verba (anuario Galego de Filoloxía). Santiago de Compostela: Universidad de Santiago de Compostela.

Ross, J. R. (1970): “On declarative sentences”, in R. A. Jacobs y P. S. Rosenbaum (eds.), Readings in English transformational grammar. Waltham, MA: Ginn and Co., pp. 222-272.

Sánchez JimÉnez, S. U. (2002): "La expresión de finalidad en la Edad Media", Dicenda 20, pp. 285-323.

SÁNCHez López, C. (1999): "La negación”, in I. Bosque y V. Demonte (coords.): Gramática descriptiva de la lengua española, II. Madrid: Espasa Calpe, pp. 2561-2634.

SweETSER, E. (1990): "Conjunction, coordination and subordination", in From etymology to pragmatics. Cambridge: Cambridge University Press, pp. 76-112. https://doi.org/10.1017/CBO9780511620904.005

Traugott, E. C. y Trousdale, G. (2013): Constructionalization and Constructional Changes. Oxford: Oxford University Press. https://doi.org/10.1093/ acprof:oso/9780199679898.001.0001

Trousdale, G. (2014): "On the relationship between grammaticalization and constructionalization", Folia Linguistica 48:2, pp. 557-577. https://doi.org/10.1515/ flin. 2014.018 
